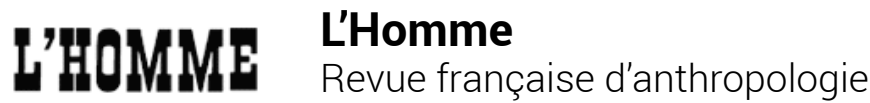

185-186 | 2008

L'anthropologue et le contemporain : autour de Marc Augé

\section{Politique et globalisation}

Perspectives anthropologiques

\section{Marc Abélès}

\section{(2) OpenEdition \\ Journals}

\section{Édition électronique}

URL : http://journals.openedition.org//homme/24131

DOI : 10.4000//homme.24131

ISSN : 1953-8103

\section{Éditeur}

Éditions de l'EHESS

\section{Édition imprimée}

Date de publication : 1 janvier 2008

Pagination : 133-143

ISSN : 0439-4216

\section{Référence électronique}

Marc Abélès, "Politique et globalisation », L'Homme [En ligne], 185-186 | 2008, mis en ligne le 01 janvier 2010, consulté le 19 avril 2019. URL : http://journals.openedition.org//homme/24131 ; DOI : 10.4000/lhomme.24131 


\title{
Politique et globalisation
}

Perspectives anthropologiques

\author{
Marc Abélès
}

\section{“}

Aujourd'hui, sous quelque aspect qu'on l'appréhende, le contexte est mondial. Le moindre campement amazonien ou africain ne peut être décrit qu'en tenant compte de sa relation, plus ou moins lâche, plus ou moins efficace ou dérisoire, mais jamais sans effet à court et à long terme, au reste du monde" (Augé 2003 : 129). Si je cite ces quelques lignes, c'est qu'elles me semblent résumer le défi qui s'ouvre désormais à l'anthropologie. L'approche du contemporain en anthropologie dont Marc Augé s'est fait le pionnier et qui a permis à cette discipline d'ouvrir de nouveaux et féconds chantiers, ne peut rester indifférente à ce qu'il désigne lui-même comme un renversement de perspective. Nous vivons désormais dans un univers où la liaison entre le local et global est une donnée incontournable du présent. On ne saurait minimiser les conséquences culturelles de cette situation, quelle que soit la pertinence des interrogations sur le caractère " inédit " de la globalisation.

Il n'est en effet pas douteux que si l'extension des relations commerciales et l'internationalisation progressive des économies sont des processus qui relèvent de la longue durée, la globalisation néolibérale est un phénomène relativement récent qui a trouvé sa pleine expression dans les années 1990. Il résulte de la convergence de plusieurs facteurs différents. Le triomphe du postfordisme et les innovations technologiques de la "société des réseaux" ont rétréci la planète. Mais la globalisation est aussi inséparable de la dynamique créée et entretenue par l'objectif de performance financière. Cette dernière exigence s’impose de manière tyrannique aux acteurs industriels et commerciaux. Ce sont les critères néolibéraux qui commandent les politiques d'ajustement structurel imposées aux économies des pays en développement. Ici aussi, on est 
rentré dans l'économie planétaire, et c'est cette perception d'une mondialisation partagée (imposée) qui produit la conscience d'une appartenance au global et qui, plus que tout autre critère, permet d'identifier la globalisation. "Les gens et les lieux de par le monde sont aujourd'hui extensivement et densément connectés les uns aux autres en raison des flux transnationaux croissants de capital et de marchandises, d'informations et d'idées, et d'êtres humains» (Kalb $2000: 1$ ).

Le concept de globalisation a émergé dans le débat sur l'intégration croissante de l'économie mondiale. Peu ou prou, lorsqu'on évoque la globalisation, reviennent les idées d'interdépendance accélérée, de rétrécissement du monde (érosion des frontières et des barrières géographiques aux activités socio-économiques), d'action à distance, de compression de l'espace et du temps. Il est question d'intégration globale, de recomposition des relations de pouvoir interrégionales, de prise de conscience d'une appartenance globale. Les contributions théoriques les plus marquantes sur ce thème se distinguent par l'importance qu'elles accordent aux données matérielles et économiques ou aux modes de représentation des formes spatio-temporelles de la mondialisation. Il est clair en tout cas que l'usage du concept de la globalisation renvoie à une perception de la mondialisation qui met l'accent sur les changements d'échelle, la magnitude croissante, l'accélération, l'impact plus intense des flux et des modèles d'interaction sociale interrégionaux.

La perspective économique n'épuise pas, bien entendu, la question de la globalisation. Aussi ne s'étonnera-t-on pas que les anthropologues aient porté leurs investigations sur ses conséquences culturelles et mis l'accent sur trois thématiques qui permettent de mieux les cerner. Ils se sont intéressés d'une part à la thématique des migrations, volontaires ou forcées, d'autre part aux identités et surtout aux formes de recompositions identitaires induites par l'intensification des flux d'humains et de marchandises et l'interconnexion croissante entre des univers de sens éloignés et hétérogènes. À ces deux thèmes s'en ajoute un troisième qui concerne le rapport entre le local et le global. Certes, les méthodes des anthropologues les prédisposent à privilégier le micro et le local ; or, de plus en plus, l'articulation du micro et du macro, du local et du global devient un élément essentiel de l'analyse. D'où les efforts mis en œuvre pour repenser la notion de "terrain » et les propositions relatives au développement d'une ethnographie "multi-site» (Marcus 1998) et à la redéfinition des rapports entre l'anthropologue et ses interlocuteurs. On peut considérer de ce point de vue que la prise en compte de la globalisation, et les débats et controverses qu'elle suscite constituent un remarquable stimulant heuristique pour l'anthropologie. Reste cependant une question qui mérite d'être 
abordée frontalement, même si elle est au cœur de cette problématique : il s'agit de la dimension politique de la globalisation.

Depuis une vingtaine d'années, l'État-nation se trouve au centre de tous les débats sur la globalisation. L'une des questions qui revient sans cesse concerne le destin de l'État-nation. Les adeptes des thèses globalistes voient dans sa fragilisation un trait marquant de la mutation qui s'est amorcée à la fin du XXe siècle. Leurs adversaires s'en prennent à cette thèse décliniste et clament haut et fort que la forme étatique, loin d'être affaiblie, a encore de beaux jours devant elle et peut devenir l'instrument efficace des nouvelles dynamiques transnationales. Pour saisir la portée de ce débat, il faut avoir à l'esprit qu'il met en présence, de chaque côté, des théoriciens et des praticiens de la politique dont le discours se présente non seulement comme un constat, mais aussi sur le mode prédictif, chacun ayant en vue l'évolution de l'état de choses existant et la perspective d'une amélioration des dispositifs institutionnels.

Face à cette approche dont j'essaierai de restituer brièvement les principaux attendus, comment se situe l'anthropologie? Cette dernière n'a pas attendu le défi du global pour développer une réflexion qui prend en compte d'autres paradigmes possibles que celui de l'État-nation. On peut même affirmer que ce qui a fait l'originalité de l'ethnographie politique, c'est sa propension à révéler l'existence de systèmes qui n'avaient rien de commun avec notre tradition occidentale. Depuis le XIX siècle, les sociétés "sans État» sont au centre de ses investigations. Autant dire que l'anthropologie n'est pas la moins bien armée pour prendre au sérieux les mutations qui affectent aujourd'hui le champ politique. Elle nous permet en effet d'échapper à l'obsession de l'État qui oriente la plupart des débats sur la globalisation.

Que nous disent les politistes? Ils observent que l'interdépendance entre économies, la nécessité d'un changement d'échelle dans la gestion des flux financiers et humains, a pour effet de fragiliser la notion de souveraineté. Bien sûr, il existe encore des frontières, des délimitations qui permettent de distinguer dans leur pluralité les États-nations. De même, comme en témoigne éloquemment l'actualité des conflits sur la planète, la revendication du plein exercice de la souveraineté demeure omniprésente un peu partout. Mais la politique des grandes puissances, qui avait atteint son apogée avec la Guerre froide et qui s’est concrétisée dans un véritable ordre mondial polarisé autour du clivage entre Est et Ouest, laisse place à une réalité plus complexe marquée par un double processus. D'un côté, une apparente hégémonie de l'hyper-puissance, les États-Unis; de l'autre, la perception d'une fragilité de ce pouvoir organisé face à une violence dont l'événement du 11 septembre a été la manifestation la plus spectaculaire. 
L'un des constats les plus généralement partagés concernant les conséquences de la globalisation souligne l'exacerbation des inégalités, notamment entre le Nord et le Sud, et la déstructuration des tissus sociaux et culturels traditionnels. Dans ce contexte, l'apparition de nouvelles formes de violence fondées sur le sacrifice de soi qui peut prendre des formes plus ou moins sophistiquées a modifié la donne classique de la polémologie. Face à la violence d'État, c'est l'action de réseaux déterritorialisés, mobiles, fragmentés, qui tend désormais à prévaloir. De quoi déstabiliser les stratégies longtemps prévalentes qui mettaient au premier plan un type d'organisation centralisée et qui reposaient en grande partie sur les performances des industries d'armement.

Par ailleurs, de nouvelles formes d'organisations jouent un rôle politique de premier plan dans des domaines aussi divers que l'environnement, l'économie, l'humanitaire. Elles présentent une fluidité, une souplesse, qui contraste avec les structures rigides des appareils étatiques traditionnels. Les organisations non gouvernementales (ONG) se développent aux quatre coins du monde, souvent en liaison avec les situations de crise, et dessinent un nouveau modèle politique plus directement ancré dans la société civile et qui transcende allègrement les frontières nationales. La transnationalité qui caractérise de plus en plus l'univers mondialisé impose de nouvelles solidarités en réseau, des modes d'action plus labiles. On peut voir ainsi d'ores et déjà se dessiner des souverainetés postnationales (Pandolfi 2002), et l'idée même de patriotisme ne perd pas toute valeur dans la mesure où on aurait affaire à un patriotisme " pluriel, contextuel et mobile» (Appadurai $2001: 244$ ).

Les globalistes font état de l'émergence d'une « gouvernance sans gouvernement " (Rosenau \& Czempiel 1992) dans un "monde sans souveraineté " (Badie 1999). Dans une perspective analogue, Ulrich Beck (2000) définit la globalisation comme une société sans État-monde, sans gouvernementmonde. Pour le sociologue, la nouveauté ne se situe pas dans les phénomènes d'interaction et dans la mise en place de réseaux denses et transfrontaliers, mais dans la perception de cette transnationalité et du caractère " non localisé » de la communauté. Désormais chacun est conscient de l'existence de dangers écologiques globaux, et de la réalité des relations transculturelles. Avec l'expérience de l'Union européenne, on prend aussi la mesure de l'impact des acteurs transnationaux et de leur pouvoir.

Le débat fait rage entre ceux qui décryptent dans notre présent les prémices d'une véritable gouvernance globale et leurs adversaires qui crient au mirage. Pour ces derniers, la globalisation ne fait qualimenter les illusions de ceux qui croient au déclin inéluctable des États nationaux. On opposera, certes, à cette vision le fait que les États aient consenti dans le 
cadre de la construction européenne à abandonner une part de leur souveraineté et à accepter la perspective d'une intégration dans un ensemble plus large. On invoquera aussi l'influence grandissante sur la scène globale d'organisations non étatiques et transnationales comme les ONG.

Même des globalistes convaincus, qui soutiennent comme Susan Strange (1996) que «les forces impersonnelles du marché sont plus puissantes que celles des États », ne reconnaissent pas moins que l'Étatnation est une réalité bien vivante. Que les nationalismes soient bien présents, il suffit de se référer aux guerres les plus récentes et à la symbolique qui les entoure. Le fait que le nombre d'États souverains ait quadruplé depuis 1945, et que les ancrages nationaux demeurent vivaces même au sein de l'élite capitaliste transnationale est également significatif. Dans le monde postcolonial, les États ne sont pas réductibles à des survivances condamnées à l'obsolescence. Les problèmes de frontière n'ont jamais été si présents. Même si les gens migrent d'un point à un autre, ils ne sont pas moins en quête d'un ancrage spatial, et la localisation, la délimitation d'un espace demeure un problème pertinent. D'autant que l'économie globalisée ne cesse d'entretenir les inégalités entre les territoires.

Le fait que soit aujourd'hui posée la question du rôle, voire de l'avenir, de l'État-nation est en tout cas révélateur du doute qui s'insinue à propos de la pertinence de ce qui constituait le mode d'exercice du pouvoir dans nos sociétés. Mais tout se passe comme si, en définitive, la question centrale était celle du devenir de la souveraineté dans le monde incertain où nous vivons. Une ligne de partage, au premier abord infranchissable, sépare ceux qui considèrent que l'État en tant qu'incarnation de la souveraineté demeure maître du jeu, seul porteur de la légitimité démocratique et rempart irremplaçable face aux dangers du "tout-économique », et ceux qui, à l'inverse, considèrent que son déclin est désormais inéluctable. La communauté nationale est-elle ou non destinée à jouer un rôle de figuration dans des processus qu'il lui sera de plus en plus difficile de maitriser? C'est la question qui revient de manière lancinante dans les débats entre globalistes et souverainistes. À y regarder de plus près, on peut se demander cependant si de part et d'autre, on n'a pas affaire à une conception étroite de la souveraineté. Autrement dit, la fin de la politique des États est-elle synonyme d'une disparition de toute forme de souveraineté ? À la globalisation correspond toute une série de transformations, lesquelles ont trait aux modes de régulation et de contrôle. Le changement d'échelle se traduit par une hétérogénéité des intervenants, l'affirmation des dimensions régionale et transnationale, et l'intrication de plus en plus étroite entre acteurs publics et privés. 
Il est significatif que les débats entre spécialistes soient aussi polarisés sur la question de l'État, de sa permanence ou de sa disparition annoncée. Mais ils manifestent une propension certaine à poser le problème en termes institutionnels, comme si la question essentielle était celle de la forme d'organisation et de l'involution qui l'affecterait ou non dans la période contemporaine. Ce qu'on peut surtout retenir de ces controverses, en se plaçant d'un point de vue anthropologique, c'est l'aperception confuse que quelque chose s'est déplacé dans notre appréhension de la politique. Or, ce qui est en cause est moins un type d'organisation politique qu'une certaine conception de la souveraineté qui faisait le cœur du système, aussi bien dans sa dimension nationale que dans son articulation globale. Peu importe de se demander si la globalisation a suscité ce déplacement, ou si elle n'en est que le révélateur.

Ce qui est central, c'est ce déplacement du politique qui peut ou non se traduire dans une réorganisation du champ institutionnel, mais qui de toute façon ne saurait s'y réduire. Il nous faut essayer de repérer la matière de ce déplacement et de mettre en évidence ce qui se construit dans ce processus, en termes de pratiques et de représentations politiques. Pour ce faire, la perspective anthropologique offre l'intérêt d'ouvrir sur la question plus large du rapport de l'humain au pouvoir, en offrant des termes de comparaison trop souvent ignorés ou refoulés par les discours de la modernité. Or, plus que jamais, nous avons besoin de ce détour. Qu'on ne se méprenne pas cependant: il ne s'agit pas du geste, après tout, assez arbitraire qui consisterait à substituer un point de vue à un autre, le "regard " de l'anthropologue étant censé être plus pénétrant que les autres. C’est tout simplement la nature même du déplacement, et les enjeux qu'il dessine, qui incitent à emprunter cette piste. Nous avons affaire à des processus dont la portée dépasse de très loin la dialectique dans laquelle on cherche à les enfermer. Ce qui s'invente aujourd'hui n'a que peu à voir avec les cadres institutionnels et conceptuels auxquels nous sommes accoutumés. Du point de vue de l'anthropologue, cette situation n'a rien de particulièrement choquant. C'est même l'un des apports de l'anthropologie d'avoir mis en évidence des dispositifs politiques qui ne cadraient pas avec les concepts philosophiques et politologiques auxquels nous étions accoutumés.

De même, lorsqu'ils se sont intéressés aux formes contemporaines de l'État, les anthropologues ont effectué un travail de déconstruction, soucieux de mettre en évidence les processus de réification qui s'y opèrent. Akhil Gupta et James Ferguson (2002) montrent comment la métaphore de la verticalité est constitutive de la représentation de l'État, elle assure sa spatialisation. L'idée que l'État est au-dessus des gens sur lesquels il exerce son pouvoir contribue à la naturalisation d'un décalage entre la sphère de 
ce condensé du politique et celle des autres activités sociales. L'anthropologie s'intéresse spécifiquement à l'État comme construction culturelle. Il n'est pas simplement un donné institutionnel, c'est le produit d'une croyance. L'État est toujours aussi un idéal d'État: on ne saurait donc le traiter comme une simple organisation matérielle. Il est un résultat, un développement de l'activité des hommes. C'est l'idéal d'État qui flotte dans leur tête qui oriente leur action. L'État, défini comme un complexe d'activités solidaires, se perpétue dans cette relation idéelle, qui n'est autre que la croyance en la légalité, c'est-à-dire au caractère légitime d'un système de normes. L'un des objectifs de l'anthropologie politique, c'est l'étude des processus qui produisent cette représentation d'une rationalité et d'une légalité coextensive à l'exercice du pouvoir et aux relations qu'il induit. C'est aussi le décryptage de la part symbolique de l'art de gouverner.

Parmi ces croyances : l'idée que les citoyens sont traités «indifféremment" par l'État, que les mêmes règles s'appliquent à tous de manière équivalente, que son action se concrétise dans le travail statistique, et en particulier le recensement. Or, comme on a pu le montrer dans des contextes bien distincts, tels que l'Inde (Appadurai 2001), l'ex-Yougoslavie (Gossiaux 2002) ou la France (Desrosières 1990), entre l'idéal d'équivalence abstraite (pour l'État tous les citoyens se valent) et la réalité des procédures où l'on tient soigneusement compte des qualités concrètes des individus (leurs origines, leur appartenances ethniques) il y a un véritable écart. Une autre idée qui contribue à renforcer le poids de l'État, est celle qui a trait à son caractère national. Les historiens nous éclairent sur la manière dont ont été produites ces " communautés imaginées ». Là encore, on appréhende le décalage existant entre la représentation de l'État comme une organisation "naturelle" de la société et la réalité complexe des processus qui contribuent à unifier au niveau territorial et linguistique des populations le plus souvent hétérogènes. On fait appel à des mythes fondateurs, à des figures héroïsées, pour produire cette représentation d'un tout englobant et homogène. La territorialisation, l'autochtonie, l'enracinement (Detienne 2003) alimentent le récit dominant transmis par l'appareil scolaire sous le titre d'histoire nationale. On ne s'étonnera pas que la construction culturelle de l'État soit au cour des préoccupations des anthropologues : les travaux consacrés aux manifestations plurielles des mémoires de guerre dans les rituels, les formes muséales et la littérature orale en offrent des illustrations significatives. De même, l'étude des rituels du pouvoir permet de mieux comprendre ce qui est en jeu non seulement dans les manifestations cérémonielles, mais dans ces aspects cycliques de l'activité politique que constituent les campagnes électorales et les rites pratiqués par les gouvernants nationaux et locaux. 
L'État est simultanément fonctionnel et fétiche et c'est cette double propriété qui assure sa prégnance sur toutes les autres formes institutionnelles dans le champ politique. Quand les contradictions s'exacerbent entre l'universalisme revendiqué par l'État et ses serviteurs, et le fait qu'il ne représente pas au même titre tous ceux dont il se réclame, alors ce travail de déconstruction de l'imaginaire de l'État s'avère encore plus indispensable. D'où l'intérêt de problématiser cette dimension qui a été trop sous-estimée par les spécialistes, politologues et juristes. Dans le contexte de la globalisation, on assiste à une fragilisation non seulement de la puissance étatique, par le jeu conjugué du marché et des organisations transnationale, mais aussi des représentations qui s'attachent à l'État. Le topos de la verticalité se trouve ébranlé par la prolifération d'instances qui se déploient dans un espace transversal et interconnecté. Ce n'est pas tant la question du déclin de l'État qui est désormais posée que celle de sa banalisation, de sa pluralisation dans un contexte où s'imposent de nouvelles formes de gouvernementalité. L'État n'est plus dans une position de surplomb : il suffit pour en faire le constat, de prendre l'exemple des politiques d'ajustement structurel où c'est le Fonds monétaire international qui mène la danse et impose des objectifs auxquels doivent se plier des gouvernements sous peine d'être voués à la faillite économique. De même, la nécessité d'accepter la régulation d'un organisme comme l'Organisation mondiale du commerce apparaît pour beaucoup comme un moindre mal, alors qu'à défaut, c'est l'arbitraire du marché qui risque de déstabiliser bien plus profondément les économies nationales.

L'un des objectifs de l'anthropologie politique est de rendre compte des effets de la globalisation sur le fonctionnement des institutions et des organisations qui gouvernent l'économie et la société. Le transnationalisme n'est pas seulement une caractéristique du capitalisme contemporain, il conditionne également les relations de pouvoir et les référents culturels. L'une des tâches de l'anthropologie politique consiste à rendre compte des effets de la mondialisation sur le fonctionnement des organisations qui gouvernent l'économie et la société. On peut prendre comme exemple de ces nouvelles configurations institutionnelles supranationales le cas de l'Union européenne. S’y trouvent rassemblés des représentants de cultures et de traditions politiques différentes qui travaillent à l'harmonisation des législations et à la construction d'un projet englobant. Cette configuration pose plusieurs questions à l'anthropologue : elles concernent les effets de cette confrontation permanente entre des identités différentes, entre des langages et des traditions administratives hétérogènes dans une entreprise politique commune et les effets pratiques et symboliques de la déterritorialisation et du changement d'échelle dans ces nouveaux lieux de pouvoir. 
En explorant les nouveaux lieux de pouvoir transnationaux, l'anthropologie aborde en fait une question complexe, celle du déplacement du politique. L'analyse en termes d'institutions et d'acteurs ne rend qu'imparfaitement compte de ce qui bouge en profondeur depuis une quinzaine d'années. La globalisation suscite un changement profond de nos rapports au politique. Il se caractérise par la montée en puissance d'une représentation qui met les préoccupations du vivre et du survivre au cœur de l'agir politique, alors que la problématique de la convivance qui focalise la cité et le rapport entre l'individu et la souveraineté est rejetée au second plan (Abélès 2006). La question de la survivance prend évidemment d'autant plus de relief qu'elle correspond à l'affaissement de l'État-providence. Le thème du développement durable et la place prise par les ONG s'inscrivent dans cette représentation d'un monde marqué par la menace et le risque, où régulièrement des événements tragiques viennent rappeler aux humains leur précarité. L'activité des ONG est centrée sur l'économie de la survie et leur discours qui porte la marque de l'urgence s'inscrit aussi dans l'horizon d'un avenir rendu incertain par les menaces qu'induit la marchandisation de l'univers. De même, la place qu'occupent aujourd'hui des institutions transnationales comme l'OMC, l'ONU et le FMI ne tient pas seulement à leur fonctionnalité dans le système global. Si l'on peut parler d'une gouvernementalité globale, à propos de cette constellation d'institutions et d'organisations transnationales, ce n'est pas dans le sens d'un réordonnancement inclusif, comme d'un pouvoir qui se superposerait aux pouvoirs existants. On assiste en fait à une reconfiguration du politique qui, pas plus qu'elle n'implique l'extinction de l'État-nation, ne s'accommode d'un aménagement de la gouvernance exclusivement centré sur la territorialité. Cet espace du global-politique s'est densifié en internalisant en quelque sorte l'angoisse anthropologique face aux menaces en tout genre, du terrorisme à la vulnérabilité écologique de la planète, alors que la forme État-nation n'était pas en mesure de donner une réponse satisfaisante à la question des droits de l'homme. Le global-politique nous projette dans un régime d'anticipation et porte le signe de l'incomplétude. Il ne peut pas être circonscrit en termes de rapport de forces ni pensé comme une forme superétatique. Il se construit, dans une certaine mesure, à côté de l'univers des partages territoriaux, sans s'imposer aux États et aux dispositifs classiques de la souveraineté comme un pouvoir surplombant. Aujourd'hui, le globalpolitique se trouve en grande partie dans la dépendance des stratégies étatiques nationales. Mais, comme le montre l'impact des initiatives qui ont trait à l'économie de la survie, il impose son régime propre et met sous pression des pouvoirs qui ne le maitrisent qu'imparfaitement. Le global-politique contamine la souveraineté ; ainsi peut-on interpréter la manière dont s'imposent des thématiques portées par les ONG. 
«Le nouvel espace-temps de notre histoire planétaire est en voie de constitution. Comme toujours c'est une constitution qui exprime des rapports de force et de pouvoir mais elle a ses contradictions particulières ", écrit Marc Augé (2003 : 161). C'est précisément l'une des tâches stimulantes qui s'ouvre à l'anthropologie que de viser à rendre compte de ce jeu complexe des espaces, des pouvoirs et des temporalités dans le contexte de la globalisation.

École des hautes études en sciences sociales, Paris Laboratoire d'anthropologie des institutions et organisations sociales (LAIOS), IIAC marc.abeles@ehess.fr

MOTS CLÉS/KEYWORDS: mondialisation/globalization - politique/politics - nord-sud/northsouth relations - survie/survival - postcolonialisme/postcolonial studies. 
Abélès, Marc

2006 Politique de la survie. Paris,

Flammarion.

Appadurai, Arjun

2001 Après le colonialisme. Les conséquences culturelles de la globalisation. Paris, Payot.

Augé, Marc

2003 Pour quoi vivons-nous? Paris, Fayard.

\section{Badie, Bertrand}

1999 Un monde sans souveraineté. Paris, Fayard.

Beck, Ulrich

2000 "What is Globalization?", in David Held \& Anthony Mc Grew, eds, The Global Transformations Reader. An Introduction to the Globolisation Debate. Lanham, Rowman \& Littlefield Publishers : 99-105.

\section{Desrosières, Alain}

1990 "How to Make things together : Social Science, Statistics and the State" in Pete Wagner et al., eds, Discourses on Society. The Shaping of the Social Science Disciplines. Boston, Kluwer Academic Publishers : 200-221.

\section{Detienne, Marcel}

2003 Comment être autochtone. Du pur Athénien au Français raciné. Paris, Le Seuil.

\section{Gossiaux, Jean-François}

2002 Problèmes ethniques dans les Balkans.

Paris, PUF.

\section{Gupta, Akhil \& James Ferguson}

2002 « Spatializing States: Toward an Ethnography of Neoliberal Governmentality ", American Ethnologist 29 (4) : 981-1002.

Kalb, Don

2000 «Localizing Flows : Power, Paths, Institutions and Networks ", in Don Kalb, et al., eds, The Ends of Globalization.

Bringing Society Back. Lanham, Rowman \& Littlefield.

\section{Marcus, George E.}

1998 Ethnography through Thick and Thin. Princeton, Princeton University Press.

\section{Pandolfi, Mariella}

2002 “"Moral entrepreneurs", souverainetés mouvantes et barbelés ", Anthropologie et Sociétés 26 (1) : 29-51.

\section{Rosenau, James \& Ernst-Otto Czempiel} 1992 Governance without Government. Order and Change in World Politics.

Cambridge, Cambridge University Press.

\section{Strange, Susan}

1996 The Retreat of the State. The Diffusion of Power in World Economy. Cambridge, Cambridge University Press. 